\title{
Three-dimensional intramolecular dynamics: Internal rotation of $\left(\mathrm{CH}_{3}\right)_{3} \mathrm{GeBr}$
}

\author{
Melanie Schnell \\ Fritz-Haber-Institut der Max-Planck-Gesellschaft, Faradayweg 4-6, D-14195 Berlin, \\ Germany \\ Jens-Uwe Grabow \\ Gottfried-Wilhelm-Leibniz-Universität Hannover, Institut für Physikalische Chemie, \\ Lehrgebiet A, Callinstr. 3-3a, D-30167 Hannover, Germany
}

\begin{abstract}
The microwave spectra of $\left(\mathrm{CH}_{3}\right)_{3}{ }^{74} \mathrm{Ge}^{79} \mathrm{Br}$ and its isotopologues $\left(\mathrm{CH}_{3}\right)_{3}{ }^{72} \mathrm{Ge}{ }^{79} \mathrm{Br}$ and $\left(\mathrm{CH}_{3}\right)_{3}{ }^{74} \mathrm{Ge}^{81} \mathrm{Br}$ have been studied in the frequency range from $2.4-20 \mathrm{GHz}$ revealing the complex internal dynamics of this organometallic molecule with three internal rotors. The assignment of the complex spectrum has been facilitated by permutation-inversion theory - the appropriate molecular symmetry group is $G_{162}$. The $V_{3}$ barrier to internal rotation is determined to be $4.783(12) \mathrm{kJ} / \mathrm{mol}$. An analysis of the bromine quadrupole coupling yields the description of the $\mathrm{Ge}-\mathrm{Br}$ and the $\mathrm{Ge}-\mathrm{C}$ bonding characters. From this analysis we find that the bromine atom has a positive partial charge resulting from $\pi$-backbonding of the bromine towards germanium. From isotopic substitution, the $\mathrm{Ge}-\mathrm{Br}$ bond distance could be determined to $2.34589(21) \AA$.
\end{abstract}

Key words:

PACS:

\section{Introduction}

Trimethylgermanium bromide, $\left(\mathrm{CH}_{3}\right)_{3} \mathrm{GeBr}$, is a organometallic symmetric top with $\mathrm{C}_{3 v}$ symmetry in its equilibrium geometry. The rotational spectra of such molecules are especially interesting since they can show complicated

Email address: schnell@fhi-berlin.mpg.de (Melanie Schnell). 
splitting patterns arising from three chemically equivalent methyl tops, or, from a group-theoretical point of view, splittings from three $C_{3 v}$-equivalent, individually $C_{3 v}$-symmetric rotors. From these splittings not only information on the torsional barrier height but also on torsion-torsion interaction terms, i.e., on top-top communication, can be obtained. For their analysis, group-theoretical considerations are a prerequisite to understand the very complex line patterns. In the past only few spectroscopic studies on molecules of the type $\left(\mathrm{CH}_{3}\right)_{3} \mathrm{XY}$ have been reported in which the torsionrotation splitting is resolved $[1,2,3,4,5,6]$.

In general, both steric repulsion and properties of the chemical bonds are responsible for the barrier height to internal rotation, and their relative contributions in varying molecular situations are usually different. We recently presented a comparative study of the different origins of the barriers to internal rotation for the series $\left(\mathrm{CH}_{3}\right)_{3} \mathrm{XCl}$ with $\mathrm{X}=\mathrm{C}, \mathrm{Si}, \mathrm{Ge}, \mathrm{Sn}$ [4], since, although belonging to the same main group in the periodic table, these four elements are chemically quite different. The study aimed at a better understanding of the influence of the covalent radius and the properties of the central atom on the internal rotation barrier and on the top-top communication. There, we found a dramatic change in the molecular internal dynamics behavior when going from Ge to $\mathrm{Sn}$ as central atoms. From this study, not only the significantly reduced electrostatic repulsion, but also a change of the bonding situation for $\mathrm{SnC}$ bonds, which have an increased ionic contribution from $\left(\mathrm{CH}_{3}\right)_{3} \mathrm{SiCl}$ via $\left(\mathrm{CH}_{3}\right)_{3} \mathrm{GeCl}$ to $\left(\mathrm{CH}_{3}\right)_{3} \mathrm{SnCl}$, can be identified as being responsible.

As a complementary study on molecular internal dynamics, we decided to keep the central atom but exchange the halogen substituent, to investigate its effect on the torsional barrier, which, again, can be attributed to both steric arguments and a change in the properties of the chemical bond. We recently recorded and analysed the high-resolution microwave spectrum of $\left(\mathrm{CH}_{3}\right)_{3} \mathrm{GeCl}$ including hyperfine structure and torsional splittings [3]. The barrier height is determined to be $V_{3}=372.359(47) \mathrm{cm}^{-1}\left(\left(\mathrm{CH}_{3}\right)_{3}{ }^{74} \mathrm{Ge}^{35} \mathrm{Cl}\right)$. The Ge-C bond character was found to be dominated by a $\sigma$ single bond ( 81 $\%)$ with a smaller ionic contribution (19\%). The Ge-Cl bond, on the other hand, has mainly $\sigma$ single bond (46.5\%) and ionic character (37.6\%), and $15.9 \% \pi$ double bond contributions [4].

Here, we present an analysis of the torsion-rotation spectrum of $\left(\mathrm{CH}_{3}\right)_{3} \mathrm{GeBr}$, i.e., we exchanged the chlorine substituent (covalence radius $99 \mathrm{pm}$ ) with the larger bromine atom (covalence radius $114 \mathrm{pm}$ ). Since, according to Pauling, the electronegativities of chlorine and bromine are very similar (3.16 and 2.98, respectively), we mainly expect a change in the molecular internal dynamics behavior due to a different steric situation. Another significant difference between chlorine and bromine as substituent is the 
nuclear quadrupole moment coupling: Both nuclei have a quadrupole spin of $\frac{3}{2}$, but the nuclear quadrupole moment coupling constant for bromine is about 8 times larger than the one for chlorine $\left(+33.1(4) \mathrm{Q} / \mathrm{fm}^{2}\right.$ for ${ }^{79} \mathrm{Br}$ and $-8.11(8) \mathrm{Q} / \mathrm{fm}^{2}$ for ${ }^{35} \mathrm{Cl}$, respectively [7]). In this study, we are also interested if this significantly larger nuclear quadrupole coupling in the presence of large amplitude motions can also lift spectral degeneracies of some hyperfine patterns as observed in $\left(\mathrm{CH}_{3}\right)_{3} \mathrm{SnCl}$, where the nuclear quadrupole coupling is small but the torsional barrier is lower and thus very dominant torsional coupling is present [4].

The rotational spectrum of $\left(\mathrm{CH}_{3}\right)_{3} \mathrm{GeBr}$ has been investigated before [8] in a higher frequency range $(26.5-40 \mathrm{GHz})$, but without resolving the bromine nuclear quadrupole coupling and the torsional fine structure, so that no information about the torsional barrier could be obtained. Furthermore, the structure of $\left(\mathrm{CH}_{3}\right)_{3} \mathrm{GeBr}$ has been studied using electron diffraction and $a b$ initio calculations [9].

The remaining parts of the paper are divided as follows: In section 2 we describe the experimental details while in section 3 the observed rotational spectrum is discussed from a more qualitative point of view. In section 4 the group-theoretical aspects are described, which are necessary for the analysis of the complex torsion-rotation spectrum. In section 5 the results of the spectroscopic investigation are discussed. Section 6 concludes the paper and gives an outlook.

\section{Experimental}

$\left(\mathrm{CH}_{3}\right)_{3} \mathrm{GeBr}$, with a stated purity of $98 \%$, was purchased from Sigma-Aldrich and was used without further purification. $\left(\mathrm{CH}_{3}\right)_{3} \mathrm{GeBr}$ is a liquid at room temperature with a boiling point of $114^{\circ} \mathrm{C}$ and a melting point of $-25^{\circ} \mathrm{C}$. Because of its relatively high vapor pressure a carrier gas was flowed through a reservoir filled with $\left(\mathrm{CH}_{3}\right)_{3} \mathrm{GeBr}$ followed by a supersonic expansion through a pulsed nozzle into the microwave resonator. A broadband $(2-26.5 \mathrm{GHz})$ high resolution Fourier transform microwave (FTMW) spectrometer molecular beam excitation apparatus, shown in Figure 1 including the CAESAR arrangement (coaxially aligned electrodes for Stark effect applied in resonators) [10], was used to observe the rotational spectrum of $\left(\mathrm{CH}_{3}\right)_{3} \mathrm{GeBr}$. The coaxially oriented beam resonator arrangement (COBRA) [11] results in line wid ths of about $1 \mathrm{kHz}$ (half width at half height $(\mathrm{HWHH})$ ) for argon and about $1.5 \mathrm{kHz}(\mathrm{HWHH})$ for neon as carrier gas. This yields a resolving power of about $3 \mathrm{kHz}$ for argon and about $5 \mathrm{kHz}$ for neon, respectively. The spectrometer was designed to provide high sensitivity and resolution also in the low frequency range below $6 \mathrm{GHz}$, which 


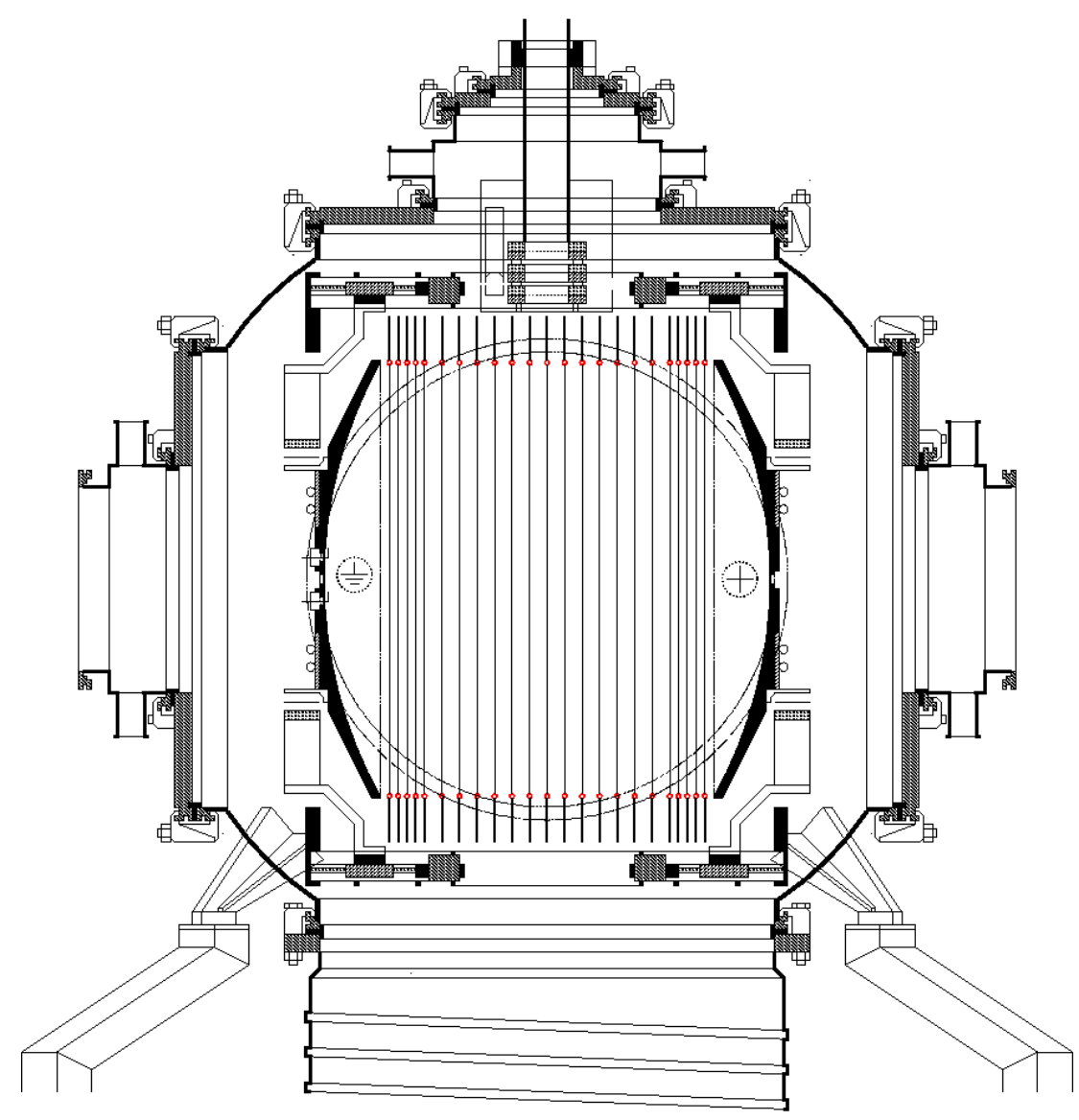

Figure 1. Experimental setup of the molecular beam excitation FTMW spectrometer including an assembly for Stark-effect measurements. The molecules are supersonically expanded into the resonator through a pulsed nozzle located in the front reflector (COBRA arrangement). The microwave antennae and the pulsed supersonic-jet source are mounted on the same reflector. For Stark-effect measurements the rear reflector is raised to a high voltage with respect to the front reflector kept at ground potential. Additional ring electrodes between the two reflectors insure a homogeneous electric field along the resonator axis (CAESAR arrangement).

was particularly helpful for this study since the $J+1 \leftarrow J=1 \leftarrow 0$-transition of $\left(\mathrm{CH}_{3}\right)_{3} \mathrm{GeBr}$ lies near $2.4 \mathrm{GHz}$. In this study, four rotational transitions, namely the $J+1 \leftarrow J=1 \leftarrow 0-, 3 \leftarrow 2-, 4 \leftarrow 3$-, and the $7 \leftarrow 6$-transitions, have been measured.

\section{Geometry considerations and spectral observations}

$\left(\mathrm{CH}_{3}\right)_{3} \mathrm{GeBr}$ has $\mathrm{C}_{3 v}$-symmetry in its equilibrium configuration, which is displayed in Figure 2. Consequently, its rotational spectrum should follow that for a prolate symmetric top. 


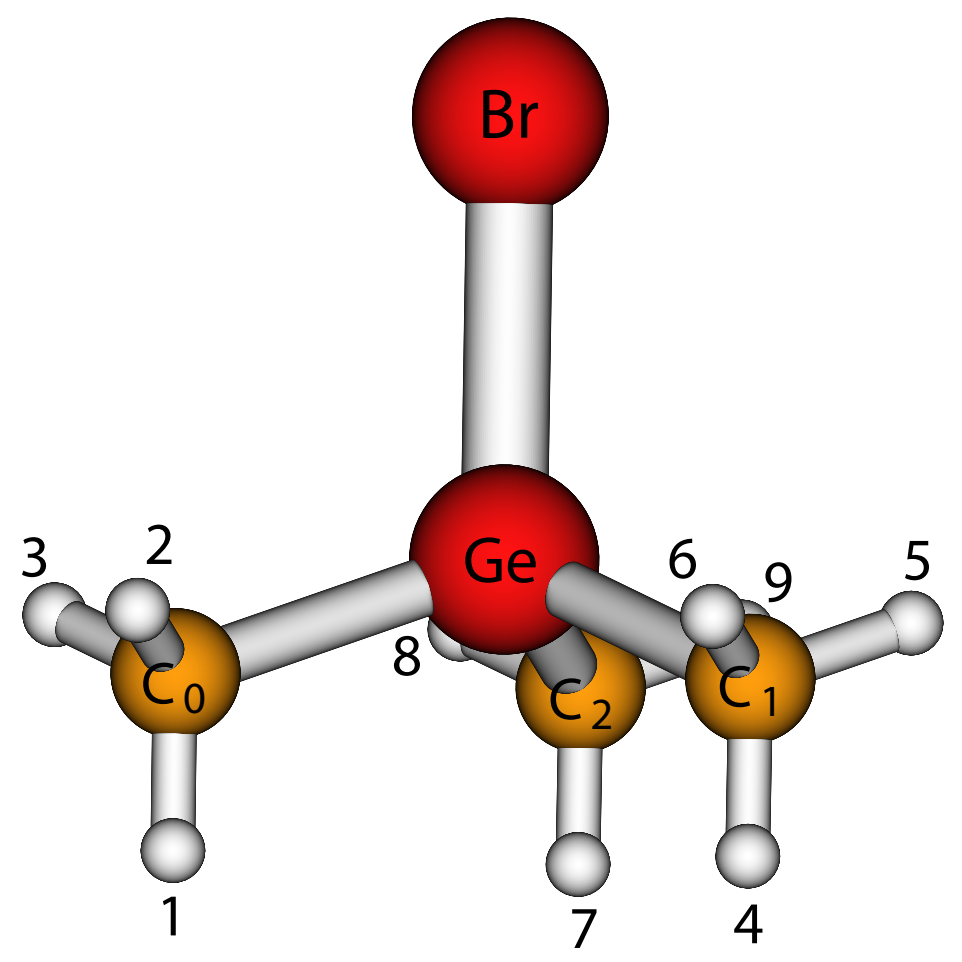

Figure 2. Geometry and numbering of the identical nuclei of $\left(\mathrm{CH}_{3}\right)_{3} \mathrm{GeBr}$. This configuration corresponds to framework 1 in the high-barrier torsion-rotation formalism described in section 4.2.

The observed rotational spectrum is complicated by rather dense line patterns due to internal rotation of the three methyl tops, bromine nuclear quadrupole hyperfine structure, and the large number of isotopes of germanium and bromine. Based on our results for $\left(\mathrm{CH}_{3}\right)_{3} \mathrm{GeCl}$ [3] we estimate a torsional barrier of about $4.5 \mathrm{~kJ} / \mathrm{mol}\left(370 \mathrm{~cm}^{-1}\right)$ for one methyl group.

Figure 3 shows a scheme of the different splitting patterns and subgroups observed for $\left(\mathrm{CH}_{3}\right)_{3} \mathrm{GeBr}$, with the displayed splitting hierarchy being valid for rotational transitions up to the $J+1 \leftarrow J=4 \leftarrow 3$-transition. For the highest transition measured in this study, $J+1 \leftarrow J=7 \leftarrow 6$, the fine structure due to internal rotation exhibits larger splittings than the hyperfine structure originating from the bromine nuclear quadrupole coupling.

One rotational $J+1 \leftarrow J$-transition can first be divided into two subgroups, one for the ${ }^{79} \mathrm{Br}$ - and one for the ${ }^{81} \mathrm{Br}$-isotopologue (Figure 3). They are further split due to the five germanium isotopologues. This can be nicely seen from 
Figure 4 , which shows a survey spectrum of the $J+1 \leftarrow J=7 \leftarrow 6$ rotational transition. The isotopologues corresponding to ${ }^{81} \mathrm{Br}$ are located at the lowerfrequency end of the spectrum, while the ones for ${ }^{79} \mathrm{Br}$ are at the higherfrequency end. For the $J+1 \leftarrow J=7 \leftarrow 6$-transition the two subgroups do not overlap. The intensities of the survey spectrum also resemble nicely the natural relative abundances of the various germanium species $\left({ }^{76} \mathrm{Ge}(7.67 \%\right.$ natural relative abundance), $\left.{ }^{74} \mathrm{Ge}(36.74 \%),{ }^{72} \mathrm{Ge}(27.37 \%),{ }^{70} \mathrm{Ge}(20.55 \%)\right)$, with ${ }^{73} \mathrm{Ge}(7.67 \%)$ as an exception. Its nuclear spin of $I\left({ }^{73} \mathrm{Ge}\right)=\frac{9}{2}$ results in a very complex quadrupole splitting and thus in a decreased intensity. Each of these groups are now further split due to internal rotation of the methyl groups and quadrupole coupling due to the bromine nucleus $\left(I(\mathrm{Br})=\frac{3}{2}\right)$. In total, one single $J+1 \leftarrow J$-symmetric top rotational transition with $J>0$ shows more than 1000 lines in the spectrum.

Figure 5 shows the amplitude spectrum of two closely lying $K=0$ bromine nuclear quadrupole components $\left(F^{\prime} \leftarrow F=\frac{11}{2} \leftarrow \frac{9}{2}\right.$ and $\left.F^{\prime} \leftarrow F=\frac{9}{2} \leftarrow \frac{7}{2}\right)$ of the $\left(\mathrm{CH}_{3}\right)_{3}{ }^{74} \mathrm{Ge}^{81} \mathrm{Br}$ isotopologue for the $J+1 \leftarrow J=4 \leftarrow 3$-transition. The different torsion-rotation components can be nicely distinguished. Each line is split into two Doppler components, which result from the coaxial beam resonator arrangement. The assignment of the different torsion-rotation symmetry species and nuclear quadrupole coupling hyperfine components is described in detail in section 4.2.

\section{Group Theory / Theoretical Considerations}

\subsection{The group $G_{162}$}

To facilitate the assignment and the fitting of spectroscopic constants to this complex rotational spectrum we used permutation-inversion (PI) group theory. The PI group $\mathrm{G}_{162}$, appropriate for $\left(\mathrm{CH}_{3}\right)_{3} \mathrm{GeBr}$, as well as statistical weights for the torsional species have already been worked out $[12,13,14]$. With the internal rotation of the three methyl groups considered feasible, the resulting molecular symmetry (MS) group $\mathrm{G}_{162}$ (Table 1 ) is formed from a direct product of three $C_{3}$-groups $\left(C_{3}\left(C_{0}\right) \otimes C_{3}\left(C_{1}\right) \otimes C_{3}\left(C_{2}\right)=G_{27}\right)$ for the three methyl groups following the numbering in Figure 2 and a semi-direct product with the $C_{3 v}$-group (six elements) for the equilibrium symmetry [14]. It consists of 13 classes. With the numbering of the hydrogen and carbon nuclei given in Figure 2 the generating operations are $\alpha=(123), \beta=(456), \gamma=(789)$, $D=\left(C_{0}, C_{1}, C_{2}\right)(1,4,7)(2,5,8)(3,6,9)$ and $R=\left(C_{1}, C_{2}\right)(2,3)(4,7)(5,9)(6,8)^{*}$. The MS-group $\mathrm{G}_{162}$ has four doubly degenerate species $\left(E_{i}, i=1-4\right)$, three sixfold- $\left(I_{j}, j=1-3\right)$, and four threefold-degenerate irreducible representations $\left(F_{4 a}, F_{4 b}\right.$ and $F_{5 a}, F_{5 b}$, respectively). $F_{4 a}, F_{4 b}$ and $F_{5 a}, F_{5 b}$, respectively, are 


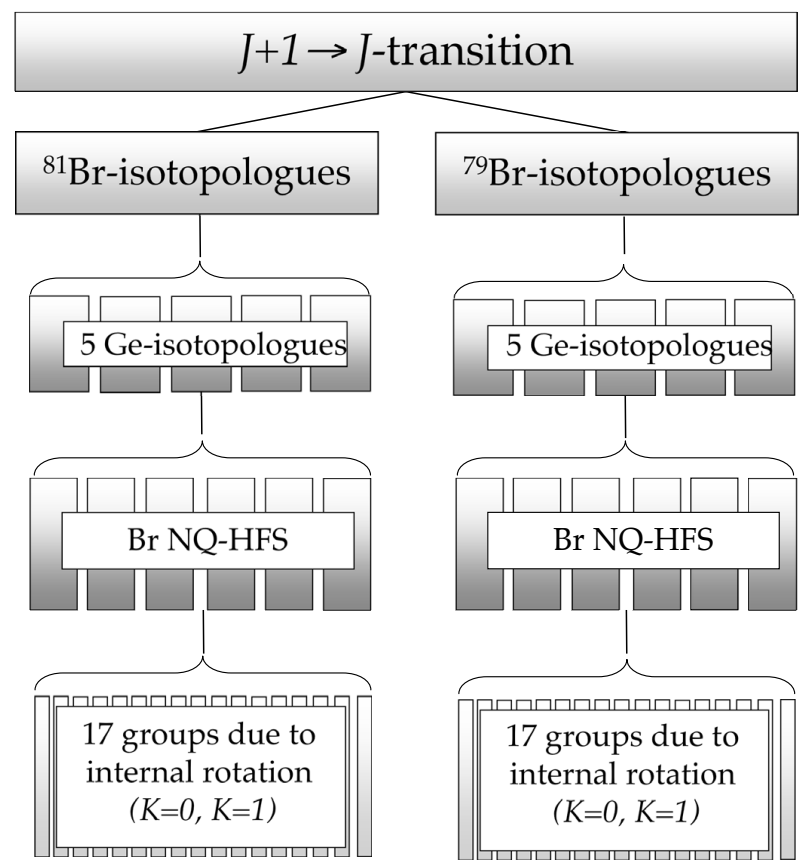

Figure 3. Schematic representation of the characteristics of the rotational spectrum of $\left(\mathrm{CH}_{3}\right)_{3} \mathrm{GeBr}$. One rotational $J+1 \leftarrow J$-transition is divided into several subgroups because of $\mathrm{Br}$ - and Ge-isotopologues, internal rotation of the three equivalent $\mathrm{CH}_{3}$ groups and $\mathrm{Br}$ nuclear quadrupole hyperfine structure. The $J+1 \leftarrow J=4 \leftarrow 3$-transition spreads over $400 \mathrm{MHz}$. It is further divided into two subgroups, one for the ${ }^{81} \mathrm{Br}$ - and one for the ${ }^{79} \mathrm{Br}$-isotopologue. They are further split due to the five germanium isotopologues, which are separated by about 8 $\mathrm{MHz}$, the bromine nuclear quadrupole coupling and internal rotation of the three identical methyl tops, the latter being the smallest splitting $(100-500 \mathrm{kHz})$.

separably degenerate because of time inversion $[15,16]$ and can be combined in pairs to give the reducible representations $I_{4}$ and $I_{5}$ [13], respectively, as shown in the last two rows of Table 1.

To derive the torsional splitting pattern for a rotational energy level the transformation properties of the rotational and other basis functions have to be determined. These transformation properties can in turn only be determined after the relation between the laboratory-fixed and molecule-fixed coordinates has been defined. In fact, the coordinates for $\left(\mathrm{CH}_{3}\right)_{3} \mathrm{GeBr}$ can be obtained from those for $\left(\mathrm{CH}_{3}\right)_{3} \mathrm{SnCl}$, which are derived in another paper 


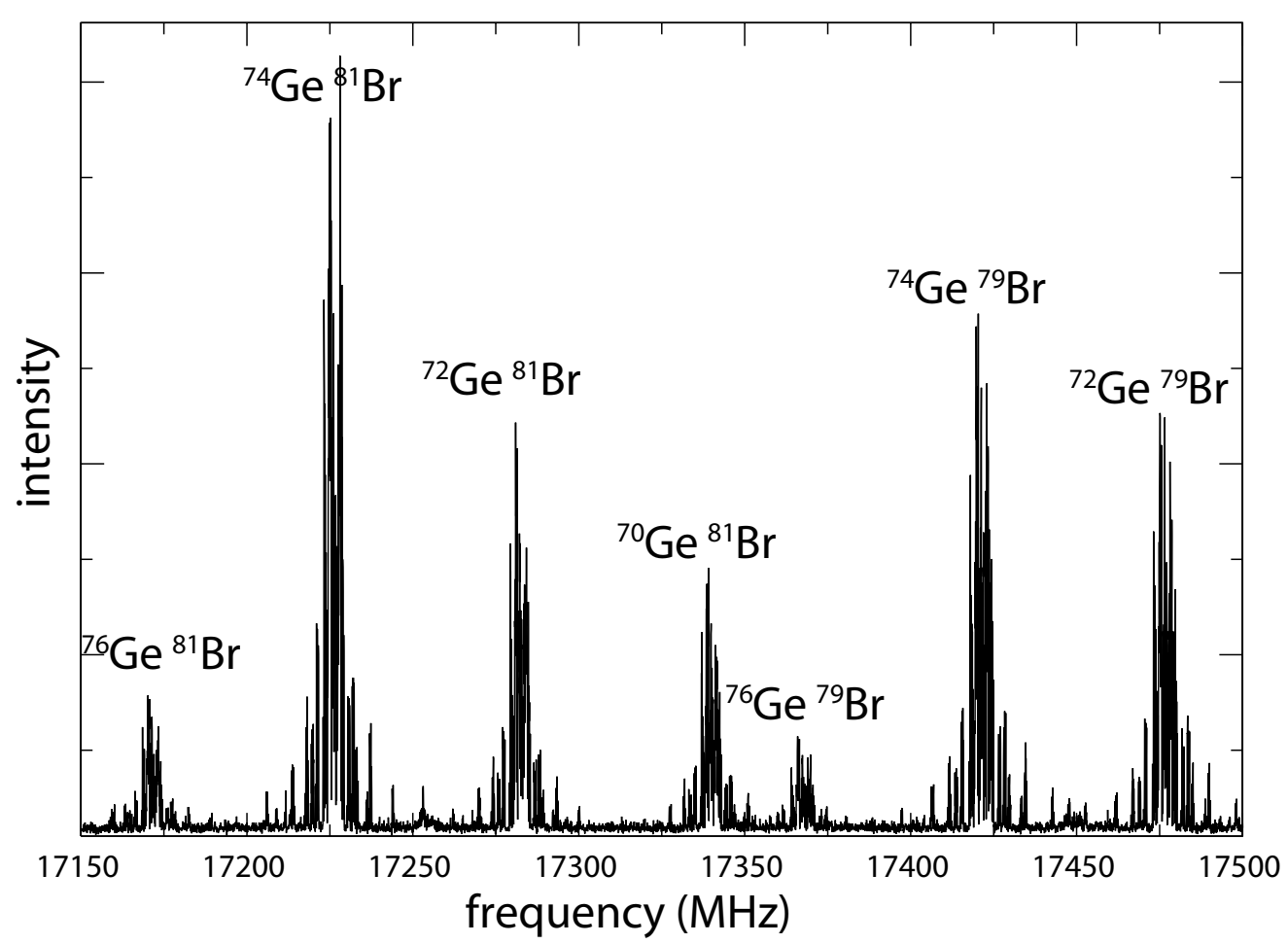

Figure 4. Survey spectrum of the $J+1 \leftarrow J=7 \leftarrow 6$ rotational transition. It spreads over about $400 \mathrm{MHz}$. The various isotopologues due to the different germanium and bromine isotopes are well separated and also the natural abundances are nicely resembled. One group for a given isotopologue is further divided due to internal rotation and bromine nuclear quadrupole coupling.

(section 4.2., equ. 1-8 of Ref. [17]), by replacing the Sn atom with the Ge atom, and using the appropriate bond lengths. Here, we only give the resulting transformation properties of the nine independent variables $\mathbf{R}, \alpha_{0}, \alpha_{1}, \alpha_{2}, \chi$, $\theta$, and $\phi$ (Table 2). $\mathbf{R}$ are the coordinates for the molecular center-of-mass, $\alpha_{0}, \alpha_{1}, \alpha_{2}$ are the torsional angles for the $\mathrm{CH}_{3}$ groups 0,1 , and 2 , respectively, corresponding to the labeling in Figure 2. $\chi, \theta$, and $\phi$ are the Eulerian angles as defined in Ref. [18], appendix 1.

Based on these transformation properties the determination of the symmetry species of the different rotational and torsional states becomes possible. The torsional states and their symmetry species are given in Table 3 . The symmetries of the symmetric top wavefunctions $|J K M\rangle$ are $A_{1}$ and $A_{2}$ for $J$ even and odd, respectively, when $K=3 n, n \geq 0$, and $E_{1}$ when $K=3 n \pm 1$.

\subsection{High-barrier tunneling-rotation formalism}

To determine the relative ordering and thus to enable an assignment of the torsional transitions observed in the rotational spectrum we used the 


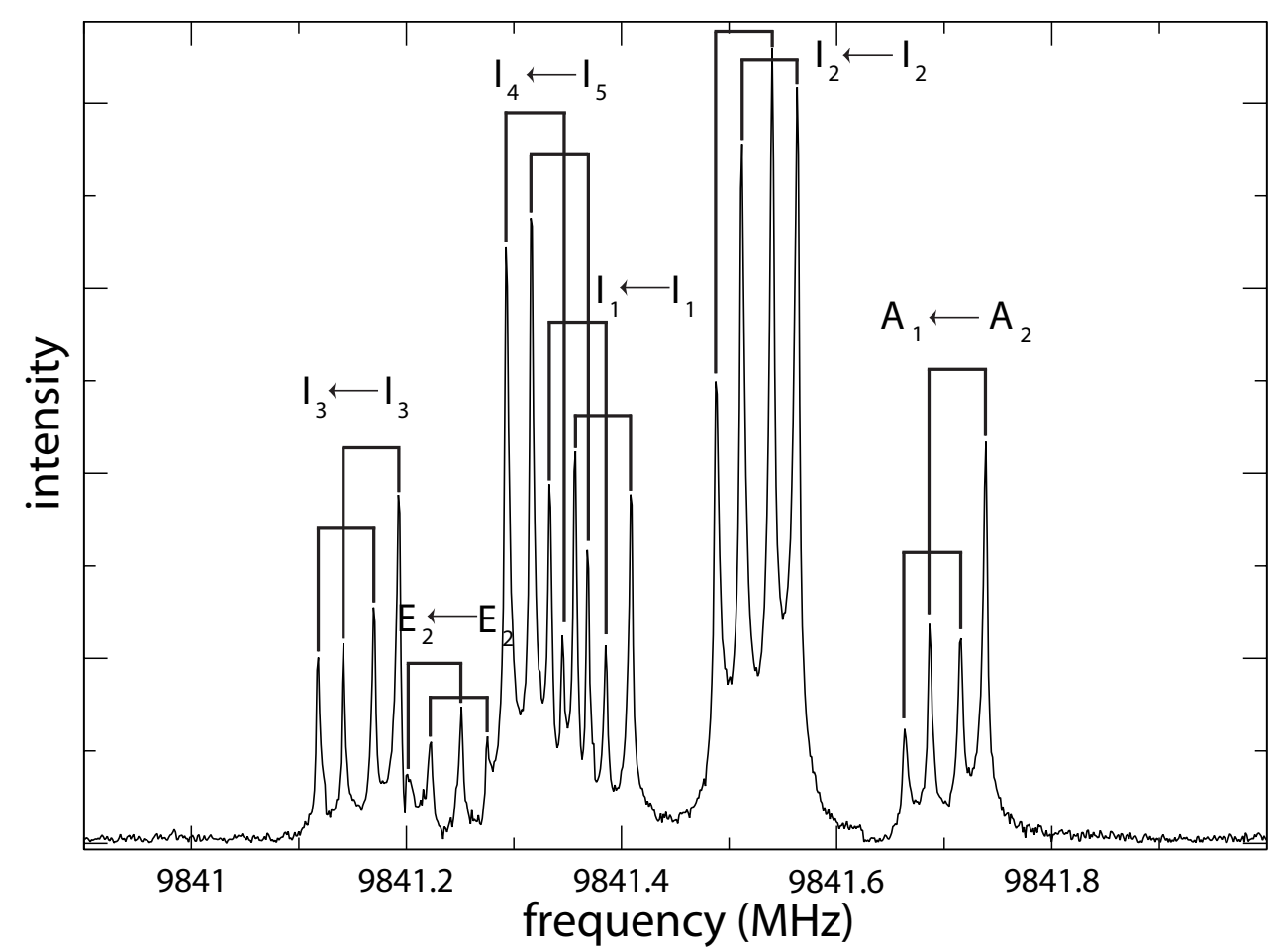

Figure 5. Amplitude spectrum of two $K=0$ bromine nuclear quadrupole components $\left(F^{\prime} \leftarrow F=\frac{11}{2} \leftarrow \frac{9}{2}\right.$ and $\left.F^{\prime} \leftarrow F=\frac{9}{2} \leftarrow \frac{7}{2}\right)$ of the $\left(\mathrm{CH}_{3}\right)_{3}{ }^{74} \mathrm{Ge}^{81} \mathrm{Br}$ isotopologue $(J+1 \leftarrow J=4 \leftarrow 3$ rotational transition). The different torsion-rotation components can be nicely distinguished. Each line is split into two Doppler components, which are marked by bars and which result from the coaxial beam resonator arrangement. For each torsion-rotation transition two $K=0$ bromine quadrupole coupling components are present, which are $23 \mathrm{kHz}$ apart.

high-barrier group-theoretical tunneling-rotation formalism [19] appropriate for $G_{162}$, based on the coordinates and their transformation properties defined above, to predict the torsional splitting pattern for $K=0$. For a $\mathrm{C}_{3 v}$-symmetric molecule with three group-theoretically equivalent internal rotors like $\left(\mathrm{CH}_{3}\right)_{3} \mathrm{GeBr} n=27$ different frameworks are possible. These can be connected through tunneling pathways represented by respective matrix elements in a tunneling Hamiltonian matrix. If, e.g., the initial framework is taken to be framework 1, which is displayed in Figure 2, then the 351 pathways and corresponding matrix elements can be denoted as framework $n \rightarrow$ framework $n^{\prime},\left(n, n^{\prime}=1-27\right)$, and $H_{n, n^{\prime}}$, respectively. However, the properties of the group $G_{162}$ show that only five of these tunneling pathways are topologically inequivalent. They correspond physically to: i) rotation of only one $\mathrm{CH}_{3}$-group $\left(\mathrm{H}_{R}=\mathrm{H}_{1, n}, n=2-7\right)$, ii) rotation of two $\mathrm{CH}_{3}$-groups in a geared $\left(H_{G}=H_{1, n}, n=14-19\right)$ or antigeared way $\left(H_{A}=H_{1, n}, n=8-13\right)$, and iii) rotation of all three $\mathrm{CH}_{3}$-groups at the same time, either having the same $\left(H_{E}=H_{1, n}, n=20,27\right)$ or opposite sense $\left(H_{L}=H_{1, n}, n=21-26\right)$. In the high-barrier tunneling-rotation formalism it is necessary to assume 
Table 1

Character Table of $\mathrm{G}_{162}$ with $\epsilon=\exp (2 \pi i / 3) . F_{4 a}$ and $F_{4 b}$ as well as $F_{5 a}$ and $F_{5 b}$ are separably degenerate to each other and can be combined to the reducible representations $I_{4}$ and $I_{5}$ as given in the last two rows.

\begin{tabular}{ccccccccccccccc}
\hline \hline & $\mathrm{E}$ & $\alpha \beta \gamma$ & $\alpha \beta^{-1}$ & $\alpha^{-1} \beta$ & $\alpha$ & $\alpha \beta$ & $\alpha^{-1} \beta \gamma$ & $\mathrm{D}$ & $\alpha \mathrm{D}$ & $\alpha^{-1} \mathrm{D}$ & $\mathrm{R}$ & $\beta \mathrm{R}$ & $\beta^{-1} \mathrm{R}$ & \\
& 1 & 2 & 3 & 3 & 6 & 6 & 6 & 18 & 18 & 18 & 27 & 27 & 27 & \\
\hline $\mathrm{A}_{1}$ & 1 & 1 & 1 & 1 & 1 & 1 & 1 & 1 & 1 & 1 & 1 & 1 & 1 & $\mathrm{~T}_{z}($ even $)$ \\
$\mathrm{A}_{1}$ & 1 & 1 & 1 & 1 & 1 & 1 & 1 & 1 & 1 & 1 & -1 & -1 & -1 & $\mathrm{R}_{z}(\mathrm{odd})$ \\
$\mathrm{E}_{1}$ & 2 & 2 & 2 & 2 & 2 & 2 & 2 & -1 & -1 & -1 & 0 & 0 & 0 & $\left(\mathrm{~T}_{x}, \mathrm{~T}_{y}\right)\left(\mathrm{R}_{x}, \mathrm{R}_{y}\right)$ \\
$\mathrm{E}_{2}$ & 2 & 2 & 2 & 2 & -1 & -1 & -1 & 2 & -1 & -1 & 0 & 0 & 0 & \\
$\mathrm{E}_{3}$ & 2 & 2 & 2 & 2 & -1 & -1 & -1 & -1 & 2 & -1 & 0 & 0 & 0 & \\
$\mathrm{E}_{4}$ & 2 & 2 & 2 & 2 & -1 & -1 & -1 & -1 & -1 & 2 & 0 & 0 & 0 & \\
$\mathrm{I}_{1}$ & 6 & -3 & 0 & 0 & 0 & -3 & 3 & 0 & 0 & 0 & 0 & 0 & 0 & \\
$\mathrm{I}_{2}$ & 6 & -3 & 0 & 0 & 3 & 0 & -3 & 0 & 0 & 0 & 0 & 0 & 0 & \\
$\mathrm{I}_{3}$ & 6 & -3 & 0 & 0 & -3 & 3 & 0 & 0 & 0 & 0 & 0 & 0 & 0 & \\
$\mathrm{~F}_{4 a}$ & 3 & 3 & $3 \epsilon^{*}$ & $3 \epsilon$ & 0 & 0 & 0 & 0 & 0 & 0 & 1 & $\epsilon$ & $\epsilon^{*}$ & \\
$\mathrm{~F}_{4 b}$ & 3 & 3 & $3 \epsilon$ & $3 \epsilon^{*}$ & 0 & 0 & 0 & 0 & 0 & 0 & 1 & $\epsilon^{*}$ & $\epsilon$ & \\
$\mathrm{F}_{5 a}$ & 3 & 3 & $3 \epsilon^{*}$ & $3 \epsilon$ & 0 & 0 & 0 & 0 & 0 & 0 & -1 & $-\epsilon$ & $-\epsilon^{*}$ & \\
$\mathrm{~F}_{5 b}$ & 3 & 3 & $3 \epsilon$ & $3 \epsilon^{*}$ & 0 & 0 & 0 & 0 & 0 & 0 & -1 & $-\epsilon^{*}$ & $-\epsilon$ & \\
$\mathrm{I}_{4}$ & 6 & 6 & -3 & -3 & 0 & 0 & 0 & 0 & 0 & 0 & 2 & -1 & -1 & \\
$\mathrm{I}_{5}$ & 6 & 6 & -3 & -3 & 0 & 0 & 0 & 0 & 0 & 0 & -2 & 1 & 1 & \\
\hline \hline
\end{tabular}

Table 2

Transformation properties of the nine variables of $\left(\mathrm{CH}_{3}\right)_{3} \mathrm{GeBr}$ under the generating operations of $\mathrm{G}_{162}$.

\begin{tabular}{lccccc}
\hline & $\mathbf{R}$ & $\alpha_{0}$ & $\alpha_{1}$ & $\alpha_{2}$ & rotation \\
\hline$(123)$ & $\mathbf{R}$ & $\alpha_{0}+2 \pi / 3$ & $\alpha_{1}$ & $\alpha_{2}$ & $\chi, \theta, \phi$ \\
$(456)$ & $\mathbf{R}$ & $\alpha_{0}$ & $\alpha_{1}+2 \pi / 3$ & $\alpha_{2}$ & $\chi, \theta, \phi$ \\
$(789)$ & $\mathbf{R}$ & $\alpha_{0}$ & $\alpha_{1}$ & $\alpha_{2}+2 \pi / 3$ & $\chi, \theta, \phi$ \\
$\mathrm{D}$ & $\mathbf{R}$ & $\alpha_{1}$ & $\alpha_{2}$ & $\alpha_{0}$ & $\chi+2 \pi / 3, \theta, \phi$ \\
$\mathrm{R}$ & $-\mathbf{R}$ & $-\alpha_{0}$ & $-\alpha_{2}$ & $-\alpha_{1}$ & $\pi-\chi, \pi-\theta, \pi+\phi$ \\
\hline
\end{tabular}

that $\left(\mathrm{CH}_{3}\right)_{3} \mathrm{GeBr}$ spends most of its time vibrating in the vicinity of one of these 27 frameworks and only occasionally tunnels from one conformation to another [20]. Quantum mechanically speaking, we require the splittings caused by tunneling motions to be small compared to the vibrational spacings associated with a single equilibrium configuration (framework).

Based on our very rough $a b$ initio calculations the distance of closest approach between hydrogen atoms connected to neighboring methyl groups is reduced from $3.3 \AA$ in the equilibrium configuration (Figure 2) to $3.1 \AA$ for tunneling pathways with one methyl group internally rotating $\left(H_{R}\right)$, and further to $2.7 \AA$ for tunneling pathways with the two methyl groups 
Table 3

Symmetry species of the torsional $\left(\Gamma_{\text {torsional }}\right)$ and torsion-rotation components of the rotational levels $K=3 n$ and $K=3 n \pm 1(n=0,1,2, \ldots)$.

\begin{tabular}{|c|c|c|c|c|c|}
\hline \multirow{2}{*}{$\left|M_{0} M_{1} M_{2}\right\rangle$} & \multirow{2}{*}{$\Gamma_{\text {torsional }}$} & \multicolumn{2}{|c|}{$K=0$} & \multirow{2}{*}{$K=3 n \pm 1$} & \multirow{2}{*}{$K=3 n$} \\
\hline & & $J_{\text {even }}$ & $J_{o d d}$ & & \\
\hline$|0,0,0\rangle$ & $\mathrm{A}_{1}$ & $\mathrm{~A}_{1}$ & $\mathrm{~A}_{2}$ & $\mathrm{E}_{1}$ & $\mathrm{~A}_{1}+\mathrm{A}_{2}$ \\
\hline$| \pm 1,0,0\rangle$ & $\mathrm{I}_{2}$ & $\mathrm{I}_{2}$ & $\mathrm{I}_{2}$ & $2 \mathrm{I}_{2}$ & $2 \mathrm{I}_{2}$ \\
\hline$| \pm 1, \pm 1,0\rangle$ & $\mathrm{I}_{1}$ & $\mathrm{I}_{1}$ & $\mathrm{I}_{1}$ & $2 \mathrm{I}_{1}$ & $2 \mathrm{I}_{1}$ \\
\hline$| \pm 1, \mp 1,0\rangle$ & $\mathrm{I}_{4}$ & $\mathrm{I}_{4}$ & $\mathrm{I}_{5}$ & $\mathrm{I}_{4}+\mathrm{I}_{5}$ & $\mathrm{I}_{4}+\mathrm{I}_{5}$ \\
\hline$| \pm 1, \pm 1, \mp 1\rangle$ & $\mathrm{I}_{3}$ & $\mathrm{I}_{3}$ & $\mathrm{I}_{3}$ & $2 \mathrm{I}_{3}$ & $2 \mathrm{I}_{3}$ \\
\hline$| \pm 1, \pm 1, \pm 1\rangle$ & $E_{2}$ & $\mathrm{E}_{2}$ & $\mathrm{E}_{2}$ & $E_{3}+E_{4}$ & $2 \mathrm{E}_{2}$ \\
\hline
\end{tabular}

internally rotating $\left(H_{G}\right.$ and $\left.H_{A}\right)$. The van-der-Waals radius for hydrogen is $r_{H_{v d W}}=1.2 \AA$. In a simple approach $2 \cdot r_{H_{v d W}}$ gives an approximate molecular bond length. Consequently, for tunneling pathways $H_{G}$ and $H_{A}$ hydrogen atoms of different methyl groups already come so close that the electron clouds of the $\mathrm{C}-\mathrm{H}$ bonds start to repel each other resulting in significantly higher barriers for these pathways. We furthermore assume that the barriers for $H_{E}$ and $H_{L}$ are even higher yielding only small contributions to the torsion-rotation splittings in the rotational spectrum. Thus we assume large torsion-rotation splittings (low barrier) due to internal rotation of only one methyl group $\left(H_{R}\right)$, somewhat smaller frequency shifts due to internal rotation of two methyl groups at a time $\left(H_{G}\right.$ and $\left.H_{A}\right)$ and only minor shifts due to internal rotation of all three methyl groups at a time $\left(H_{E}\right.$ and $\left.H_{L}\right)$.

With these considerations a specific tunneling Hamiltonian matrix for $K=0$ connecting all 27 frameworks through the five feasible tunneling pathways is obtained. Diagonalization of this symmetric matrix leads to a characteristic torsional splitting pattern of the $K=0$ rotational energy levels displayed in Figure 6. The diagram is most easily understood from left to right, starting with a 27-fold degenerate $J_{K}=J_{0}$ rotational energy level. The first splitting is obtained when internal rotation of one methyl top at a time is considered as feasible $\left(H_{R}\right)$ leading to four torsion-rotation energy levels $A_{1}, I_{2},\left(I_{4}, I_{1}\right)$, and $\left(I_{3}, E_{2}\right)$, with $I_{4}$ and $I_{1}$ as well as $I_{3}$ and $E_{2}$ being degenerate, consistent with a local mode picture. Consideration of simultaneous internal rotation of two methyl tops $\left(H_{G}\right.$ and $H_{A}$, with $\left.H_{G}=-H_{A}\right)$ being feasible leads to a lifting of these degeneracies and to a characteristic $K=0$ torsion-rotation splitting pattern consisting of six components. The contributions for $H_{G}$ and $H_{A}$ are of the same order of magnitude, but with opposite signs. This can be explained by the orientation of the methyl groups in the equilibrium geometry (Figure 2). One hydrogen atom of every methyl group points downwards, so that starting from such a configuration both the geared and the anti-geared 
tunneling pathways have to overcome similar energy barriers.

Based on other internal rotation studies we expect the $K=0$ torsion-rotation transitions to exhibit an energy level splitting pattern of six components showing the same sequence as predicted by theory for the energy level ordering (Figure 6). We also expect the frequency ordering of the torsionrotation transitions in the spectrum to be opposite to the energy ordering of the torsion-rotation levels in Figure 6; i.e., we expect the torsion-rotation transition $A_{2} \leftarrow A_{1}\left(J_{\text {even }}\right)$ to be located at the high-frequency end of each member of a $K=0$ series, while the $A_{1}$-symmetry torsion-rotation level is located at the lowest energy in the ordering of the energy levels.

We included a section of the measured $J+1 \leftarrow J=4 \leftarrow 3$-transition $(K=0$, $F^{\prime} \leftarrow F=\frac{11}{2} \leftarrow \frac{9}{2}$ and $F^{\prime} \leftarrow F=\frac{9}{2} \leftarrow \frac{7}{2}$ ) (between $9841.0 \mathrm{MHz}$ to $9841.8 \mathrm{MHz}$ ) at the right side of Figure 6 . In order to obtain semiquantitative agreement between the theoretically derived energy level splitting pattern and the observed spectrum displayed in Figure 6, the contributions of the five different tunneling pathways have to be chosen as follows:

$$
H_{R}>-H_{A} \approx H_{G}>H_{E} \approx H_{L}
$$

This is in agreement with the beforementioned assumptions based on the distances of closest approach between the hydrogen atoms of neighboring methyl groups.

\section{Spectral analyses and discussion}

In the course of the spectral analysis we performed fits of the rotational spectra of the three most abundant isotopologues $\left(\mathrm{CH}_{3}\right)_{3}{ }^{74} \mathrm{Ge}^{81} \mathrm{Br},\left(\mathrm{CH}_{3}\right)_{3}{ }^{74} \mathrm{Ge}{ }^{79} \mathrm{Br}$, and $\left(\mathrm{CH}_{3}\right)_{3}{ }^{72} \mathrm{Ge}^{79} \mathrm{Br}$ using the least-squares fitting program XIAM, which also allows us to determine the torsional barrier $V_{3}$ [21] from the rotational spectra in the presence of nuclear quadrupole coupling hyperfine structure. XIAM is based on a Hamiltonian discussed in Ref. [21]. The global rotational constants $A$ and $B$, i.e., including torsion-rotation transitions of all measurable symmetry species, the centrifugal distortion constant $D_{J}$ as well as the chlorine quadrupole coupling constant $e q Q$ were determined. Due to numerous superpositions of lines and the lower intensities of transitions with $K \geq 1$ the fits are limited to $K=0$ transition frequencies. For the rotational constant of the methyl group $F_{0}$ a reasonable value corresponding to $I_{\alpha}=3.20 u \cdot \AA^{2}$ was chosen and kept fixed. The $A$ rotational constants for $\left(\mathrm{CH}_{3}\right)_{3}{ }^{74} \mathrm{Ge}^{79} \mathrm{Br}$ and $\left(\mathrm{CH}_{3}\right)_{3}{ }^{72} \mathrm{Ge}^{79} \mathrm{Br}$ have been fixed to the value determined for $\left(\mathrm{CH}_{3}\right)_{3}{ }^{74} \mathrm{Ge}^{81} \mathrm{Br}$. Table 4 summarizes the results of the global fits using XIAM for the three most abundant isotopologues $\left(\mathrm{CH}_{3}\right)_{3}{ }^{74} \mathrm{Ge}^{81} \mathrm{Br}$, 


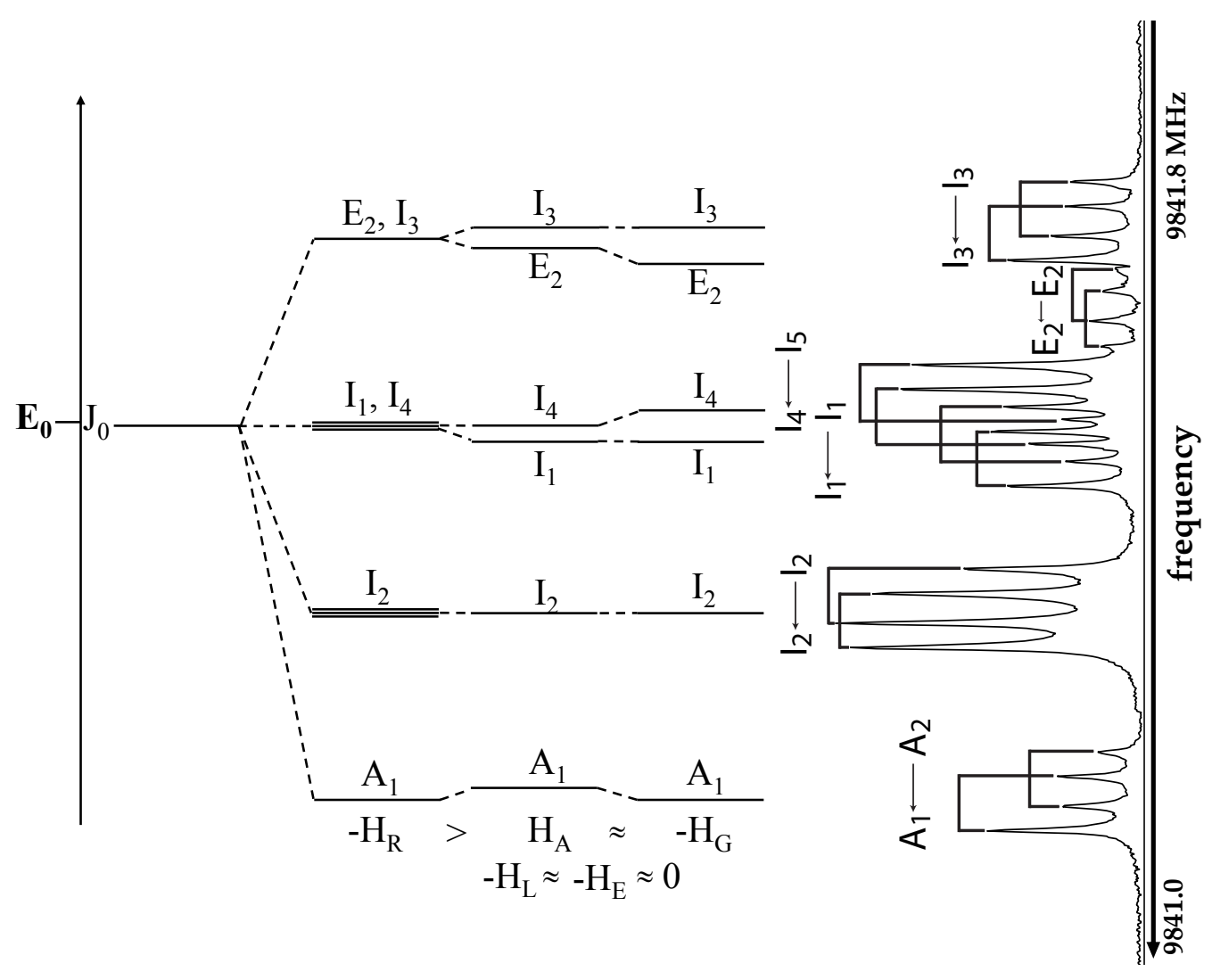

Figure 6. Torsional splitting pattern for the $J_{K}=J_{0}$ rotational energy level obtained with the high-barrier torsion-rotation tunneling formalism. The theoretical predictions are compared with the measured rotational spectrum for the $J+1 \leftarrow J=4 \leftarrow 3$-transition of the $\left(\mathrm{CH}_{3}\right)_{3}^{74} \mathrm{Ge}^{81} \mathrm{Br}$-isotopologue. For each torsion-rotation transition two lines occur due to two closely lying $K=0$ quadrupole components.

$\left(\mathrm{CH}_{3}\right)_{3}{ }^{74} \mathrm{Ge} \mathrm{e}^{79} \mathrm{Br}$, and $\left(\mathrm{CH}_{3}\right)_{3}{ }^{72} \mathrm{Ge}^{79} \mathrm{Br}$, while the observed and calculated transition frequencies are provided as supplementary data. For comparison, the rotational constants $B$ obtained by Li et al. [8] and the molecular constants for $\left(\mathrm{CH}_{3}\right)_{3}{ }^{74} \mathrm{Ge}^{35} \mathrm{Cl}[3]$ are also given.

As can be seen, the rotational constants $B$ determined in this study agree well with the ones obtained in an earlier study with lower resolution [8]. The ratio of the bromine quadrupole coupling constants of $\left(\mathrm{CH}_{3}\right)_{3}{ }^{74} \mathrm{GeBr}$ is $\mathrm{eqQ}\left({ }^{79} \mathrm{Br}\right) / \mathrm{eqQ}\left({ }^{81} \mathrm{Br}\right)=1.201$, in very good agreement with the ratio of the nuclear quadrupole moments, $\mathrm{Q}\left({ }^{79} \mathrm{Br}\right) / \mathrm{Q}\left({ }^{81} \mathrm{Br}\right)=1.199$ [7], obtained from the hyperfine structure of atomic spectra. Additionally, the bromine quadrupole coupling constants in $\left(\mathrm{CH}_{3}\right)_{3}{ }^{74} \mathrm{Ge}^{79} \mathrm{Br}$ and $\left(\mathrm{CH}_{3}\right)_{3}{ }^{72} \mathrm{Ge}^{79} \mathrm{Br}$ are, as expected, very similar.

The $V_{3}$ barrier to internal rotation is $4.783(12) \mathrm{kJ} / \mathrm{mol}$ for the $\left(\mathrm{CH}_{3}\right)_{3}{ }^{74} \mathrm{Ge}^{81} \mathrm{Br}$ isotopologue, which is similar for the other two isotopologues. As a comparison, the $V_{3}$-barrier for $\left(\mathrm{CH}_{3}\right)_{3}{ }^{74} \mathrm{Ge}^{35} \mathrm{Cl}$ is somewhat lower with 
Table 4

Molecular constants of the isotopologues $\left(\mathrm{CH}_{3}\right)_{3}{ }^{74} \mathrm{Ge}^{81} \mathrm{Br},\left(\mathrm{CH}_{3}\right)_{3}{ }^{74} \mathrm{Ge}^{79} \mathrm{Br}$, and $\left(\mathrm{CH}_{3}\right)_{3}{ }^{72} \mathrm{Ge}^{79} \mathrm{Br}$ derived from a least-square fit of the $K=0$ torsion-rotation transitions using XIAM. $F_{0}$ is the rotational constant of the methyl group corresponding to $I_{\alpha}=3.20 \mathrm{u} \cdot \AA^{2}$ and has been kept fixed during the fitting procedure, $\mathrm{N}$ is the number of lines included in the fit, and $\sigma$ gives the standard deviation of the fit. $\theta$ describes the angle between the internal rotor axis and the $a$ principal inertia axis which coincides with the $\mathrm{Ge}-\mathrm{Br}$ bond. The $A$ rotational constants for $\left(\mathrm{CH}_{3}\right)_{3}{ }^{74} \mathrm{Ge}{ }^{79} \mathrm{Br}$ and $\left(\mathrm{CH}_{3}\right)_{3}{ }^{72} \mathrm{Ge}^{79} \mathrm{Br}$ have been fixed to the value determined for $\left(\mathrm{CH}_{3}\right)_{3}{ }^{74} \mathrm{Ge}^{81} \mathrm{Br}$. For comparison, the $B$ rotational constants determined by [8] and the molecular constants for $\left(\mathrm{CH}_{3}\right)_{3}{ }^{74} \mathrm{Ge}^{35} \mathrm{Cl}[3]$ are also given.

\begin{tabular}{ccccc}
\hline & $\left(\mathrm{CH}_{3}\right)_{3}{ }^{74} \mathrm{Ge}^{81} \mathrm{Br}$ & $\left(\mathrm{CH}_{3}\right)_{3}{ }^{74} \mathrm{Ge}^{79} \mathrm{Br}$ & $\left(\mathrm{CH}_{3}\right)_{3}{ }^{72} \mathrm{Ge}^{79} \mathrm{Br}$ & $\left(\mathrm{CH}_{3}\right)_{3}{ }^{74} \mathrm{Ge}^{35} \mathrm{Cl}[3]$ \\
\hline $\mathrm{A} / \mathrm{MHz}$ & $2730.92(14)$ & {$[2730.92]$} & {$[2730.92]$} & $2875.1(21)$ \\
$\mathrm{B} / \mathrm{MHz}$ & $1230.38601(47)$ & $1244.32608(72)$ & $1248.28048(96)$ & $1989.548968(92)$ \\
$\mathrm{B} / \mathrm{MHz}[8]$ & 1230.388 & 1244.333 & 1248.268 & \\
$\mathrm{D}_{J} / \mathrm{kHz}$ & $-1.1954(48)$ & $-1.366(13)$ & $-1.290(16)$ & $0.3260(14)$ \\
$\mathrm{eQq} / \mathrm{MHz}$ & $263.368(58)$ & $316.309(64)$ & $317.234(54)$ & $-40.0703(16)$ \\
$\mathrm{V}_{3} / \mathrm{kJ} / \mathrm{mol}$ & $4.783(12)$ & $4.720(15)$ & $4.681(16)$ & $4.45441(56)$ \\
$F_{0} / \mathrm{GHz}$ & {$[158.0]$} & {$[158.0]$} & {$[158.0]$} & {$[158.0]$} \\
$\theta /{ }^{\circ}$ & {$[106.2]$} & {$[106.2]$} & {$[106.2]$} & {$[106.2]$} \\
$\mathrm{N}$ & 47 & 64 & 57 & \\
$\sigma / \mathrm{kHz}$ & 31.1 & 66.1 & 80.0 & \\
\hline
\end{tabular}

4.45441(56) $\mathrm{kJ} / \mathrm{mol}$. As can be seen from the chemical bonding analysis below, the increase in torsional barrier for $\left(\mathrm{CH}_{3}\right)_{3} \mathrm{GeBr}$ compared to $\left(\mathrm{CH}_{3}\right)_{3} \mathrm{GeCl}$ is mainly due to larger electrostatic repulsion because of the larger bromine atom. A similar trend can also be extracted from two subsequent microwave studies of Merke et al. on $\left(\mathrm{CH}_{3}\right)_{3} \mathrm{SiCl}$ [1] and $\left(\mathrm{CH}_{3}\right)_{3} \mathrm{SiI}$ [5]. The $\mathrm{V}_{3}$ barrier of $\left(\mathrm{CH}_{3}\right)_{3} \mathrm{SiI}$ is with $7.4151 \mathrm{~kJ} / \mathrm{mol}$ about $0.5 \mathrm{~kJ} / \mathrm{mol}$ higher than for $\left(\mathrm{CH}_{3}\right)_{3} \mathrm{SiCl}$.

An analysis of the quadrupole coupling constants following the TownesDailey model [22] yields information on the chemical character of the $\mathrm{Ge}-\mathrm{Br}$ bond. From this analysis, the chemical bond is estimated to be dominated by a covalent single $\sigma$ contribution of $62.5 \%$ along with $-6.4 \%$ ionic and $43.9 \%$ $\pi$-bonding character. The negative sign on the total ionic bond character indicates that $\pi$-backbonding of the bromine towards germanium exceeds the polarization of the $\sigma$-bond and a positive charge results on the coupling halogen. The $\mathrm{Ge}-\mathrm{Cl}$ bond in $\left(\mathrm{CH}_{3}\right)_{3} \mathrm{GeCl}$, on the other hand, has mainly $\sigma$ single bond (46.5\%) and ionic character (37.6\%), and $15.9 \% \pi$ double bond contributions [4]. 
Using Gordy's approach [23], the $\left(\mathrm{CH}_{3}\right)_{3}-\mathrm{Ge}\left(\mathrm{CH}_{3}\right)_{2} \mathrm{X}$ bond can also be evaluated from group electronegativities [24]: For $\left(\mathrm{CH}_{3}\right)_{3} \mathrm{GeCl}$, the $\mathrm{Ge}-\mathrm{C}$ bond character was found to be dominated by a $\sigma$ single bond $(81 \%)$ with a smaller ionic contribution (19\%) [4]. For $\left(\mathrm{CH}_{3}\right)_{3} \mathrm{GeBr}$, the $\mathrm{Ge}-\mathrm{C}$ bond character is estimated to differ slightly from that of $\left(\mathrm{CH}_{3}\right)_{3} \mathrm{GeCl}$, since the $\mathrm{Ge}\left(\mathrm{CH}_{3}\right)_{2} \mathrm{Br}$ group electronegativity is lowered upon substitution by the somewhat less electronegative bromine. The bond character is still dominated by a colvalent $\sigma$-bond $(76.6 \%)$ and a slightly larger ionic contribution $(23.4 \%)$. No $\pi$-backbonding is possible for the $\mathrm{CH}_{3}$-Ge bond in any of the species. A larger ionic rather than covalent bond character is likely to lower the barrier to internal rotation. However, with the $\mathrm{Ge}-\mathrm{C}$ bond still remaining similar for the chlorine and bromine species, the somewhat higher barrier to internal rotation in $\left(\mathrm{CH}_{3}\right)_{3} \mathrm{GeBr}$ might thus be attributed to hindering from an increased electrostatic repulsion caused by the larger halogen substituent.

Durig et al. [25] reported an angle $\theta=<(\mathrm{C}-\mathrm{Ge}-\mathrm{Cl})$ of $106.3 \pm 0.1^{\circ}$, which is in good agreement with the value of $106.0^{\circ}$ obtained by MP2/6-311+G(d) geometry optimizations [9]. We used a fixed value of $106.2^{\circ}$ in our XIAM fits. From the rotational constants $B$ for the $\left(\mathrm{CH}_{3}\right)_{3}{ }^{74} \mathrm{Ge}^{79} \mathrm{Br},\left(\mathrm{CH}_{3}\right)_{3}{ }^{72} \mathrm{Ge}^{79} \mathrm{Br}$, and $\left(\mathrm{CH}_{3}\right)_{3}{ }^{74} \mathrm{Ge}^{81} \mathrm{Br}$ isotopologues a $\mathrm{Ge}-\mathrm{Br}$ bond distance can be determined via isotopic substitution expressions. From these data we obtain 2.34589(21) $\AA$ compared to $2.345 \AA(\mathrm{MP} 2 / 6-311+\mathrm{G}(\mathrm{d}))$ [9]. Aarset et al. obtained a GeBr bond distance of 2.324(4) $\AA$ from electron diffraction measurements [9]. For $\left(\mathrm{CH}_{3}\right)_{3} \mathrm{GeCl}$, we found a significantly shorter $\mathrm{Ge}-\mathrm{Cl}$ bond length of 2.15198(97) $\AA$, which can be understood with the larger covalence radius of bromine.

From an analysis of the observed splitting pattern we get the following highbarrier tunneling matrix elements $H_{i}: H_{R}=-45.0 \mathrm{kHz}, H_{A}=+5.1 \mathrm{kHz}, H_{G}=-5.3$ $\mathrm{kHz}, H_{E}=0.0 \mathrm{kHz}$, and $H_{L}=-0.3 \mathrm{kHz}$, which is in line with our assumptions mentioned above. Consequently, the contributions from $H_{E}$ and $H_{L}$ can be omitted in the high-barrier tunneling analysis (Figure 6), since they are within the experimental accuracy.

One motivation of this study was to investigate the coupling between the nuclear quadrupole moment of the halogen substituent and the angular momenta of the internal rotation of the three methyl tops. In $\left(\mathrm{CH}_{3}\right)_{3} \mathrm{SnCl}$ $\left(e q Q\left({ }^{35}\right) \mathrm{Cl}=-35.776(81) \mathrm{MHz}\right)[4]$, which exhibits very low torsional barriers and thus large torsional splittings, we found a lifting of accidental spectral degeneracies of some hyperfine components in $K=0$ transitions of certain symmetry species as a group-theoretically allowed result of $K=0$ $K=1$-mixing. For $\left(\mathrm{CH}_{3}\right)_{3} \mathrm{GeCl}$, this was not observed. By exchanging the chlorine by a bromine substituent with an about 8 times larger nuclear quadrupole coupling constant, we should be able to probe even small contributions of such $\mathrm{K}=0-\mathrm{K}=1$-mixing. However, for $\left(\mathrm{CH}_{3}\right)_{3} \mathrm{GeBr}$, this effect 
was not observed.

\section{Conclusions and discussion}

The rotational spectra of several isotopologues of $\left(\mathrm{CH}_{3}\right)_{3} \mathrm{GeBr}$ have been recorded using pulsed molecular beam high-resolution microwave spectroscopy. One rotational $J+1 \leftarrow J$-transition was found to be split into several subgroups due to internal rotation motion of the three chemically and group-theoretically equivalent methyl groups, bromine nuclear quadrupole coupling and numerous bromine and germanium isotopes.

PI group-theoretical approaches using the group $G_{162}$ have been developed and used to support an assignment of the complex torsion-rotation substructure. From the analysis, information about the different feasible tunneling pathways can be gained. As a result of a global fit including all torsional transitions of the $K=0$ rotational transitions for three main isotopologues, $\left(\mathrm{CH}_{3}\right)_{3}{ }^{74} \mathrm{Ge}^{81} \mathrm{Br},\left(\mathrm{CH}_{3}\right)_{3}{ }^{74} \mathrm{Ge}^{79} \mathrm{Br}$, and $\left(\mathrm{CH}_{3}\right)_{3}{ }^{72} \mathrm{Ge}^{79} \mathrm{Br}$, we were able to determine the barrier height for $\mathrm{CH}_{3}$ internal motion with $4.783(12) \mathrm{kJ} / \mathrm{mol}$. This is somewhat higher than for the related species $\left(\mathrm{CH}_{3}\right)_{3} \mathrm{GeCl}$ and can be assumed to be mainly due to electrostatic repulsion caused by the larger bromine atom. This is in line with the larger Ge-Br distance of 2.34589(21) $\AA$ compared to 2.15198(97) $\AA$ for the $\mathrm{Ge}-\mathrm{Cl}$ bond in $\left(\mathrm{CH}_{3}\right)_{3} \mathrm{GeCl}$ and with our analysis of the $\mathrm{Ge}-\mathrm{C}$ chemical bonds both in $\left(\mathrm{CH}_{3}\right)_{3} \mathrm{GeCl}$ and $\left(\mathrm{CH}_{3}\right)_{3} \mathrm{GeBr}$.

As for the chlorine species $\left(\mathrm{CH}_{3}\right)_{3} \mathrm{GeCl}, \mathrm{K}=0$ - $K=1$-mixing effects in the nuclear quadrupole hyperfine structures are entirely missing, even for the strongly coupling bromine where small contributions can be probed. With a decrease in the barrier heights, such effects are then expected to suddenly occur in $\left(\mathrm{CH}_{3}\right)_{3} \mathrm{SnBr}$.

\section{Acknowledgements}

The authors thank Jon T. Hougen for helpful discussions. Financial support of the Land Niedersachsen and the Deutsche Forschungsgemeinschaft is gratefully acknowledged. M.S. is grateful for a Liebig-Stipendium of the Fonds der chemischen Industrie. 


\section{References}

[1] I. Merke, W. Stahl, S. Kassi, D. Petitprez, G. Wlodarczak, Internal rotation, quadrupole coupling, and structure of (ch3)3sicl studied by microwave spectroscopy and ab initio calculation, J. Mol. Spec. 216 (2002) 437-446.

[2] K. Voges, J. Gripp, H. Hartwig, H. Dreizler, Analysis of torsion in a threetop molecule. torsional barrier and moment of inertia of trimethyl ethynyl germane., Z. Naturforsch. 51a (1996) 299-305.

[3] M. Schnell, J.-U. Grabow, Internal dynamics in organometallic molecules: Rotational spectrum of $\left(\mathrm{ch}_{3}\right)_{3}$ gecl, Phys. Chem. Chem. Phys. 8 (2006) 22252231.

[4] M. Schnell, J.-U. Grabow, Multi-dimensional large-amplitude motions: Toptop communication through chemical bonds, Angew. Chem. Int. Ed. 45 (2006) 3465-3470.

[5] I. Merke, A. Lüchow, W. Stahl, Internal rotation, quadrupole coupling and structure of (ch3)3sii studied by microwave spectroscopy and ab initio calculations, J. Mol. Struct. 780-781 (2006) 295-299.

[6] K. Klatte, D. Christen, I. Merke, W. Stahl, H. Oberhammer, Gas phase structure of ruppert's reagent, cf3sime3, J. Phys. Chem. A 109 (2005) 8438-8442.

[7] I. Mills, T. Cvitas, K. Homann, N. Kallay, K. Kuchitsu, Quantities, Units and Symbols in Physical Chemistry, Blackwell Science Ltd, Oxford, 1993.

[8] Y. S. Li, J. R. Durig, Spectra and structure of organogermanes. xiii. microwave spectrum of trimethylbromogermane, Inorg. Chem. 12 (1973) 306-309.

[9] K. Aarset, E. M. Page, Molecular structures of trimethylchlorogermane, (ch3)3gecl, and trimethylbromogermane, (ch3)3gebr, obtained by gas-phase electron diffraction and theoretical calculations, J. Phys. Chem. A 108 (2004) 5474-5478.

[10] M. Schnell, D. Banser, J. U. Grabow, Coaxially aligned electrodes for starkeffect applied in resonators using a supersonic jet fourier transform microwave spectrometer, Rev. Sci. Instrum. 75 (2004) 2111-2115.

[11] J.-U. Grabow, W. Stahl, H. Dreizler, A multioctave coaxially oriented beamresonator arrangement fourier-transform microwave spectrometer, Rev. Sci. Instrum. 67 (1996) 4072-4084.

[12] K. D. Möller, H. G. Andresen, On the theory of torsional vibrations of (ch3)3-x type molecules, J. Chem. Phys. 39 (1963) 17-22.

[13] K. K. Lehmann, B. H. Pate, G162: Molecular symmetry group of t-butane and other three equivalent methyl molecules, J. Mol. Spec. 144 (1990) 443-445.

[14] P. Groner, Nonrigid symmetry groups of molecular trimers and 3-rotor molecules, Spectrochimica Acta Part A 49a (1993) 1935-1946. 
[15] G. Herzberg, Infrared and Raman Spectra of Polyatomic Molecules, Van Nostrand, Princeton, NJ, 1945.

[16] G. Herzberg, Electronic Spectra and Electronic Structure of Polyatomic Molecules, Van Nostrand, Princeton, NJ, 1966.

[17] M. Schnell, J.-U. Grabow, J. T. Hougen, Towards the complete analysis of the rotational spectrum of (ch3)3sncl, in preparation, J. Mol. Spec.

[18] E. B. Wilson, Jr., J. C. Decius, P. C. Cross, Molecular Vibrations, Dover Publications, New York, NY, USA, 1980.

[19] N. Ohashi, J. T. Hougen, Application of permutation-inversion group theory to the interpretation of the microwave absorption spectrum of dimethyl methylphosphonate, J. Mol. Spec. 211 (2002) 119-126.

[20] J. T. Hougen, A rotational hamiltonian for the ground vibrational-state of hydrazine, J. Mol. Spec. 89 (1981) 296-327.

[21] H. Hartwig, H. Dreizler, The microwave spectrum of trans-2,3-dimethyloxirane in torsional excited states, Z. Naturforsch. 51 (1996) 923-932.

[22] C. H. Townes, A. L. Schawlow, Microwave Spectroscopy, Dover Publications, New York, 1975.

[23] W. Gordy, R. L. Cook, A new method of determining electronegativity from other atomic properties, Phys. Rev. 69 (1946) 604-607.

[24] W. Gordy, R. L. Cook, Microwave Molecular Spectra, 3rd Edition, John Wiley \& Sons, New York, NY, USA, 1984.

[25] J. R. Durig, K. L. Hellams, Spectra and structure of organogermanes xviii. microwave spectrum of trimethylchlorogermane, J. Mol. Struct. 29 (1975) 349356. 